\title{
Use of Short Peripheral Intravenous Catheters: Characteristics, Management, and Outcomes Worldwide
}

\author{
Evan Alexandrou, RN, BHealth, ICU Cert, MPH, PhD*1,2,3,6, Gillian Ray-Barruel, RN, BSN, BA, ICU, Cert, PhD 3,4, \\ Peter J Carr, RN, Dip HE Nurs, H Dip A\&E Nursing, BSc, MMedSc (Health Informatics), PhD $3,4,5$, Steven A Frost, RN, ICU Cert, \\ MPH, PhD 1,2,6, Sheila Inwood, RN, CNS7 , Niall Higgins, RN, GDipeH, PhD 3,8, Frances Lin, RN, PhD', Laura Alberto, RN, BN MEd \\ Dip.Com.Sc ${ }^{3}$, Leonard Mermel, DO ScM AM FACP FIDSA FSHEA9 and Claire M Rickard, RN GradDip N(CritCare), PhD, FAHMS \\ FACN ${ }^{3,4}$, and the OMG Study Group ${ }^{10}$
}

\begin{abstract}
'Western Sydney University, Sydney, New South Wales, Australia; 2Department of Intensive Care, Liverpool Hospital, New South Wales, Sydney, New South Wales, Australia; ${ }^{3}$ Alliance for Vascular Access Teaching and Research Group, Menzies Health Institute, Griffith University, Brisbane, Queensland, Australia; ${ }^{4}$ National Centre of Research Excellence in Nursing, Griffith University, Brisbane, Queensland, Australia; ${ }^{5}$ The University of Western Australia, Perth, Western Australia, Australia; ${ }^{6}$ Centre for Applied Nursing Research \& Ingham Institute for Applied Medical Research, South Western Sydney Local Health District, South Western Sydney Clinical School, University of New South Wales, New South Wales, Australia; ${ }^{7}$ Royal Berkshire Hospital, Berkshire, England; ${ }^{8}$ Queensland University of Technology, Brisbane, Queensland, Australia; ${ }^{9}$ Rhode Island Hospital and Warren Alpert Medical School of Brown University, Providence, Rhode Island, USA; ${ }^{10}$ One Million Global Peripheral Intravenous Catheter (OMG PIVC) Study collaborators in each country (see Appendix 1).
\end{abstract}

BACKGROUND: Peripheral intravenous catheter (PIVC) use in health care is common worldwide. Failure of PIVCs is also common, resulting in premature removal and replacement.

OBJECTIVE: To investigate the characteristics, management practices, and outcomes of PIVCs internationally.

DESIGN: Cross-sectional study.

SETTING/PATIENTS: Hospitalized patients from rural, regional, and metropolitan areas internationally.

MEASUREMENTS: Hospital, device, and inserter characteristics were collected along with assessment of the catheter insertion site. PIVC use in different geographic regions was compared.

RESULTS: We reviewed 40,620 PIVCs in 51 countries. PIVCs were used primarily for intravenous medication ( $n$ $=28,571,70 \%)$ and predominantly inserted in general wards ( $n=22,167,55 \%)$. Two-thirds of all devices were placed in non-recommended sites such as the hand, wrist, or antecubital veins. Nurses inserted most PIVCs $(n=$ $28,575,71 \%)$; although there was wide regional variation ( $26 \%$ to $97 \%$ ). The prevalence of idle PIVCs was $14 \%$ ( $=5,796)$. Overall, $10 \%(n=4,204)$ of PIVCs were painful to the patient or otherwise symptomatic of phlebitis; a further $10 \%(n=3,879)$ had signs of PIVC malfunction; and $21 \%$ of PIVC dressings were suboptimal $(n=8,507)$. Over one-third of PIVCs $(n=14,787,36 \%)$ had no documented daily site assessment and half ( $n=19,768,49 \%$ ) had no documented date and time of insertion.

CONCLUSIONS: In this study, we found that many PIVCs were placed in areas of flexion, were symptomatic or idle, had suboptimal dressings, or lacked adequate documentation. This suggests inconsistency between recommended management guidelines for PIVCs and current practice. Journal of Hospital Medicine. May 30, 2018. doi: 10.12788/jhm.3039 @ 2018 Society of Hospital Medicine

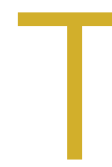

he majority of hospitalized patients worldwide have at least one peripheral intravenous catheter (PIVC), making PIVC insertion one of the most common clinical procedures. In the United States, physicians, advanced practitioners, and nurses insert over 300 million of

\footnotetext{
*Address for correspondence: Evan Alexandrou, RN BHealth ICU Cert MPH, PhD, Western Sydney University, Locked Bag 1797, Penrith South. DC 1797, New South Wales 2751, Australia; Telephone: + 6129685 9506; Fax: + 6129685 9023; E-mail: E.Alexandrou@westernsydney.edu.au

Additional Supporting Information may be found in the online version of this article.
}

Received: September 1, 2017; Revised: April 22, 2018; Accepted: April 30, 2018 2018 Society of Hospital Medicine DOI 10.12788/jhm.3039 these devices in hospitalized patients annually. ${ }^{2}$ Despite their prevalence, PIVCs are associated with high rates of complications, including insertion difficulty, phlebitis, infiltration, occlusion, dislodgment, and catheter-associated bloodstream infection (CABSI), known to increase morbidity and mortality risk. ${ }^{2-9}$ Up to $90 \%$ of PIVCs are prematurely removed owing to failure before planned replacement or before intravenous (IV) therapy completion. . $-6,10-12^{2}$

PIVC complication and failure commonly triggers insertion of a replacement device and can entail significant costs. ${ }^{2-4}$ One example is PIVC-related CABSI, where treatment costs have been estimated to be between US $\$ 35,000$ and US $\$ 56,000$ per patient. ${ }^{6,13}$ Another important consideration is the pain and anxiety experienced by patients who need a replacement device, particularly those with difficult vascular access, who may 
require multiple cannulation attempts to replace a PIVC. 12,14-16 In developing nations, serious adverse events related to PIVCs are even more concerning, because hospital acquired infection rates and associated mortality are nearly 20 times greater than in developed nations. ${ }^{17}$

A number of evidence-based interventions have been suggested to reduce PIVC failure rates. In addition to optimal hand hygiene when inserting or accessing a PIVC to prevent infection, ${ }^{18}$ recommended interventions include placement of the PIVC in an area of non-flexion such as the forearm to provide stability for the device and to reduce patient discomfort, securing the PIVC to reduce movement of the catheter at the insertion site and within the blood vessel, and use of occlusive dressings that reduce the risk of external contamination of the PIVC site. 11,19,20 Best practice guidelines also recommend the prompt removal of devices that are symptomatic (when phlebitis or other complications are suspected) and when the catheter is no longer required. ${ }^{21,22}$

Recent evidence has demonstrated that catheter size can have an impact on device survival rates. In adults, large-bore catheters of 18 gauge $(G)$ or higher were found to have an increased rate of thrombosis, and smaller-bore catheters of $22 \mathrm{G}$ or lower (in adults) were found to have higher rates of dislodgment and occlusion/infiltration. The catheter size recommended for adults based on the latest evidence for most clinical applications is $20 \mathrm{G}$. 3,20,23,24 In addition, the documentation of insertion, maintenance, and removal of PIVCs in the medical record is a requirement in most healthcare facilities worldwide and is recommended by best practice guidelines; however, adherence remains a challenge. ${ }^{1,19}$

The concerning prevalence of PIVC-related complications and the lack of comparative data internationally on organizational compliance with best practice guidelines formed the rationale for this study. Our study aim was to describe the insertion characteristics, management practices, and outcomes of PIVCs internationally and to compare these variables to recommended best practice.

\section{MATERIALS AND METHODS}

\section{Study Design and Participants}

In this international cross-sectional study, we recruited hospitals through professional networks, including vascular access, infection prevention, safety and quality, nursing, and hospital associations (Appendix 2). Healthcare organizations, government health departments, and intravascular device suppliers were informed of the study and requested to further disseminate information through their networks. A study website was developed, ${ }^{25}$ and social media outlets, including Twitter ${ }^{\circledR}$, Linkedln ${ }^{\circledR}$, and Facebook ${ }^{\circledR}$, were used to promote the study.

Approval was granted by the Griffith University Human Research Ethics Committee in Australia (reference number NRS/34/13/HREC). In addition, evidence of study site and local institutional review board/ethics committee approval was required prior to study commencement. Each participating site agreed to follow the study protocol and signed an authorship agreement form. No financial support was provided to any site.

Hospitalized adult and pediatric patients with a PIVC in situ on the day of the study were eligible for inclusion. Sample size was determined by local capacity. Hospitals were encouraged to audit their entire institution if possible; however, data were accepted from as little as one ward. Data collectors comprised nurses and doctors with experience in PIVC assessment. They were briefed on the study protocol and data collection forms by the local site coordinator, and they were supported by an overall global coordinator. Clinicians assessed the PIVC insertion site and accessed hospital records to collect data related to PIVC insertion, concurrent medications, and IV fluid orders. Further clarification of data was obtained if necessary by the clinicians from the patients and treating staff. No identifiable patient information was collected.

\section{Data Collection}

To assess whether clinical facilities were following best practice recommendations, the study team developed three data collection forms to collect information regarding site characteristics (site questionnaire), track participant recruitment (screening log), and collect data regarding PIVC characteristics and management practices (case report form [CRF]). All forms were internally and externally validated following a pilot study involving 14 sites in 13 countries.

The CRF included variables used to assess best practice interventions, such as catheter insertion characteristics (date and time, reason, location, profession of inserter, anatomical site of placement), catheter type (gauge, brand, and product), insertion site assessment (adverse symptoms, dressing type and integrity), and information related to the IV therapy (types of IV fluids and medications, flushing solutions). Idle PIVCs were defined as not being used for blood sampling or IV therapy in the preceding $24 \mathrm{~h}$.

Data collection forms were translated into 15 languages by professional translators and back-translated for validity. Translation of some languages included additional rigor. For example, Spanish-speaking members from the Spanish mainland as well as from South America were employed so that appropriate synonyms were used to capture local terms and practice. Three options were provided for data entry: directly into a purpose-developed electronic database (Lime Survey ${ }^{\circledR}$ Project, Hamburg, Germany); on paper, then transcribed into the survey database at a later time by the hospital site; or paper entry then sent (via email or post) to the coordinating center for data entry. Once cleaned and collated, all data were provided to each participating hospital to confirm accuracy and for site use in local quality improvement processes. Data were collected between June 1, 2014 and July 31, 2015.

\section{Statistical Analysis}

All data management was undertaken using SAS statistical software (SAS Institute Inc., Cary NC, USA). Results are presented for eight geographical regions using descriptive statistics (frequencies, percentages, and $95 \% \mathrm{Cls}$ ) for the variables of interest. To assess trends in catheter dwell time and rates of 
TABLE. Demographics and Characteristics by Geographical Region

\begin{tabular}{|c|c|c|c|c|c|c|c|c|c|}
\hline & \multicolumn{9}{|c|}{ Geographic Regions (n, \%) } \\
\hline & Africa & Asia & Australia / New Zealand & Europe & Middle East & North America & South America & South Pacific & Total \\
\hline & $2813(7)$ & $6428(16)$ & $5855(14)$ & $17223(42)$ & $529(1)$ & $5258(13)$ & $2384(6)$ & $130(1)$ & $40620(100)$ \\
\hline Number of Hospitals, $n(\%)$ & $49(12)$ & $30(7)$ & $82(20)$ & $150(37)$ & $6(2)$ & $56(14)$ & $32(8)$ & $1(1)$ & $406(100)$ \\
\hline Age, Median (IQR), yrs. & $45(27-64)$ & $50(32-66)$ & $63(43-77)$ & $64(43-77)$ & $44(26-65)$ & $63(47-75)$ & $46(22-64)$ & $46(22-64)$ & $59(37-74)$ \\
\hline Male gender, $\mathrm{n}(\%)$ & $1231(45)$ & $3315(52)$ & $3036(52)$ & $8889(52)$ & $279(53)$ & $2543(48)$ & $1188(50)$ & $69(53)$ & $20550(51)$ \\
\hline General ward / clinic & $1432(51)$ & $5203(81)$ & $1980(34)$ & $9596(57)$ & $304(58)$ & $2303(44)$ & $1266(54)$ & $83(64)$ & $22167(55)$ \\
\hline Emergency department & $374(13)$ & $317(5)$ & $1639(28)$ & $2958(17)$ & $134(25)$ & $1375(26)$ & $576(24)$ & $15(12)$ & $7388(18)$ \\
\hline \multicolumn{10}{|l|}{ Primary inserter, $\mathbf{n}(\%)$} \\
\hline Nurse & $1947(70)$ & $5416(84)$ & $1518(26)$ & $13524(79)$ & $506(96)$ & $3606(69)$ & $1932(81)$ & $126(97)$ & $28575(71)$ \\
\hline Doctor & $688(25)$ & $684(11)$ & $2579(45)$ & $1575(9)$ & $7(1)$ & $95(2)$ & $116(5)$ & $1(1)$ & $5745(14)$ \\
\hline \multicolumn{10}{|c|}{ Primary reason for PIVC, $\mathrm{n}(\%)$} \\
\hline \multicolumn{10}{|l|}{ Primary PIVC size, n (\%) } \\
\hline Medium (20-22 gauge) & $2042(73)$ & $3307(51)$ & $4060(69)$ & $12088(70)$ & $418(79)$ & $3887(74)$ & $1327(56)$ & $63(49)$ & $27192(67)$ \\
\hline \multicolumn{10}{|c|}{ Primary insertion site, $\mathbf{n}(\%)$} \\
\hline Hand & $1265(45)$ & $2993(47)$ & $1879(32)$ & $5017(29)$ & $295(56)$ & $1166(22)$ & $603(25)$ & $47(36)$ & $13265(33)$ \\
\hline Forearm & $798(28)$ & $1691(26)$ & $1362(23)$ & $5863(34)$ & $113(21)$ & $1937(37)$ & $863(36)$ & $48(37)$ & $12675(31)$ \\
\hline \multicolumn{10}{|c|}{ Primary PIVC dressing, $\mathbf{n}(\%)$} \\
\hline Transparent Film & $2079(74)$ & $5563(87)$ & $5677(97)$ & $11002(64)$ & $403(76)$ & $5188(99)$ & $1582(66)$ & $102(78)$ & $31596(78)$ \\
\hline
\end{tabular}

phlebitis, Poisson regression was used. All analyses were undertaken using the $\mathrm{R}$ language for statistical analysis ( $\mathrm{R}$ Core Team, Vienna, Austria). The (STROBE (Strengthening the Reporting of Observational Studies in Epidemiology statement) guidelines for cross-sectional studies were followed, and results are presented according to these recommendations. ${ }^{26}$

\section{RESULTS}

Of the 415 hospitals that participated in this study, 406 had patients with PIVCs on the day of the study (the others being small rural centers). Thus, a total of 40,620 PIVCs in 38,161 patients from 406 hospitals in 51 countries were assessed, with no more than 5\% missing data for any CRF question. There were 2459 patients $(6.1 \%)$ with two or more PIVCs concurrently in situ. The median patient age was 59 y (interquartile range [IOR], 37-74 $y)$, and just over half were male ( $n=20,550,51 \%)$. Hospital size ranged from fewer than 10 beds to over 1,000 beds, and hospitals were located in rural, regional, and metropolitan districts. The majority of countries ( $n=31,61 \%$ ) contributed multiple sites, the highest being Australia with 79 hospitals. Countries with the most PIVCs studied were Spain ( $n=5,553,14 \%)$ and the United States ( $n=5,048,12 \%)$.

General surgical ( $n=15,616,39 \%)$ and medical $(n=15,448$, $38 \%$ ) patients represented most of the population observed. PIVCs were inserted primarily in general wards or clinics ( $n=$ $22,167,55 \%$ ) or in emergency departments ( $n=7,388,18 \%$; Table) and for the administration of IV medication ( $n=28,571$, 70\%) and IV fluids ( $n=7,093,18 \%$; Table).
Globally, nurses were the primary PIVC inserters ( $n=28,575$, $71 \%$ ); however, Australia/New Zealand had only $26 \%$ ( $n=$ 1,518 ) of PIVCs inserted by this group (Table). Only about onethird of PIVCs were placed in an area of non-flexion (forearm, $n=12,675,31 \%$, Table) the majority ( $n=27,856,69 \%)$ were placed in non-recommended anatomical sites (Figure 1). Most PIVCs were placed in the hand ( $n=13,265,32.7 \%$ ) followed by the antecubital veins $(n=6176,15.2 \%)$ and the wrist $(n=5,465$, $13.5 \%$ ). Site selection varied widely across the regions; $29 \%$ ( $n$ $=1686$ ) of PIVCs in Australia/New Zealand were inserted into the antecubital veins, twice the study group average. Over half of the PIVCs inserted in the Middle East were placed in the hand ( $n=295,56 \%$ ). This region also had the highest prevalence of devices placed in nonrecommended sites ( $n=416$, 79\%; Figure 1).

The majority of PIVCs ( $n=27,192,67 \%$; Table) were of recommended size (20-22G); however, some devices were observed to be large (14-18G; $n=6,802,17 \%)$ or small (24-26g; $n=4,869,12 \%)$ in adults. In Asia, 41\% ( $n=2,617)$ of devices inserted were 24-26G, more than three times the global rate. Half of all devices in Asia $(n=3,077,48 \%)$ and the South Pacific ( $n=67,52 \%$ ) were of a size not recommended for routine IV therapy (Figure 2).

The primary dressing material used was a transparent dressing ( $n=31,596,77.8 \%$ ) (Table); however, nearly 1 in 5 dressings used had either nonsterile tape alone ( $n=5,169,13 \%$; Appendix 4), or a sterile gauze and tape ( $n=2,592,6 \%$; Appendix 4.1). We found a wide variation in the use of nonsterile tape, 


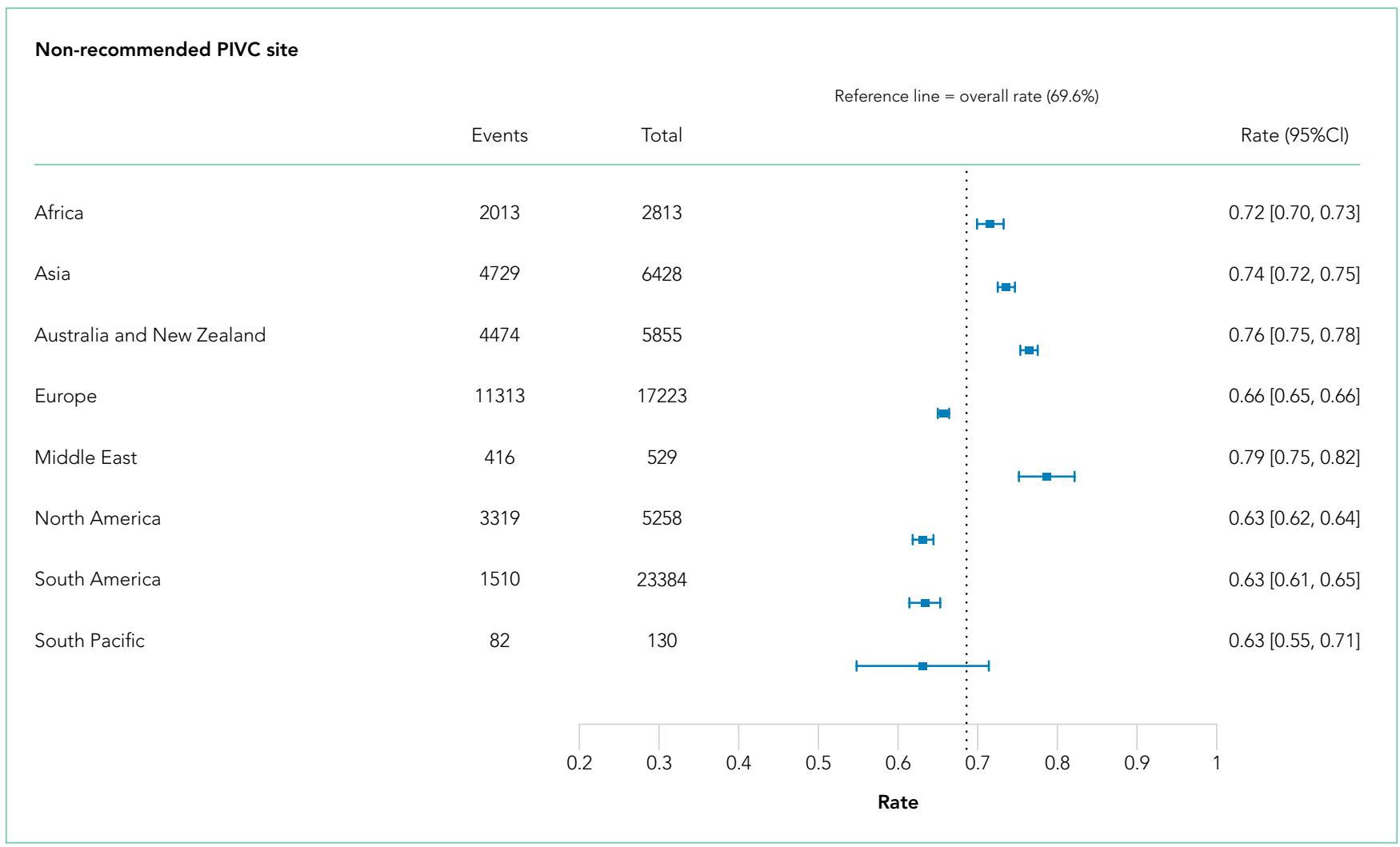

FIG 1. Non-Recommended Peripheral Intravenous Catheter site

including 1 in every 3 devices in South America dressed with nonsterile tape $(n=714,30 \%)$ and a larger proportion in Africa ( $n=543,19 \%)$ and Europe $(n=3,056,18 \%)$. Nonsterile tape was rarely used in North America and Australia/New Zealand. Although most PIVC dressings were clean, dry, and intact $(n=$ $31,786,79 \%$; Table), one-fifth overall were compromised (moist, soiled, and/or lifting off the skin). Compromised dressings (Appendix 4.2) were more prevalent in Australia/New Zealand ( $n=$ $1,448 ; 25 \%)$ and in Africa $(n=707,25 \%)$ than elsewhere.

Ten percent of PIVCs $(n=4,204)$ had signs and/or symptoms suggestive of phlebitis (characterized by pain, redness and/ or swelling at the insertion site; Appendix 4.3). The highest prevalence of phlebitis occurred in Asia $(n=1,021,16 \%)$, Africa $(n=360,13 \%)$, and South America $(n=284,12 \%)$. Pain and/ or redness were the most common phlebitis symptoms. We found no association between dwell time of PIVCs and phlebitis rates $(P=.085)$. Phlebitis rates were $12 \%$ (Days 1-3; $n=$ 15,625), 16\% (Days 4-7; $n=3,348$ ), 10\% (Days 8-21; $n=457$ ), and $13 \%$ (Day21+; $n=174)$. Nearly $10 \%(n=3,879)$ of catheters were observed to have signs of malfunction such as blood in the infusion tubing, leaking at the insertion site, or dislodgment (Appendix 4.4).

We observed $14 \%$ ( $n=5,796)$ of PIVCs to be idle (Appendix 4.5), defined as not used in the preceding $24 \mathrm{~h}$. Nearly onefourth of all devices in North America $(n=1,230,23 \%)$ and Australia/New Zealand ( $n=1,335,23 \%)$ were idle. PIVC documentation in hospital records was also poor, nearly half of all PIVCs ( $n=19,768,49 \%$ ) had no documented date and time of insertion. The poorest compliance was in Australia/New Zealand ( $n=3,428,59 \%$; Appendix 4.6). We also observed that 1 in 10 PIVCs had no documentation regarding who inserted the PIVC $(n=3,905)$. Thirty-six percent of PIVCs $(n=14,787)$ had no documented assessment of the PIVC site on the day of review (Appendix 4.7), including over half of all PIVCs in Asia ( $n$ $=3,364,52 \%$ ). Overall, the median dwell at the time of assessment for PIVCs with insertion date/time documented was 1.5 d (IQR, 1.0-2.5 d).

\section{DISCUSSION}

This international assessment of more than 40,000 PIVCs in 51 countries provides great insight into device characteristics and variation in management practices. Predominantly, PIVCs were inserted by nurses in the general ward environment for IV medication. One in ten PIVCs had at least one symptom of phlebitis, one in ten were dysfunctional, one in five PIVC dressings were compromised, and one in six PIVCs had not been used in the preceding $24 \mathrm{~h}$. Nearly half of the PIVCs audited had the insertion date and time missing.

Regional variation was found in the professions inserting PIVCs, as well as in anatomical placement. In Australia/New Zealand, the proportion of nurses inserting PIVCs was much lower than the study group average (26\% vs $71 \%$ ). Because these countries contributed a substantial number of hospitals to the study, this seems a representative finding and suggests a need for education targeted at nurses for PIVC insertion in this region. The veins in the forearm are recommended as 


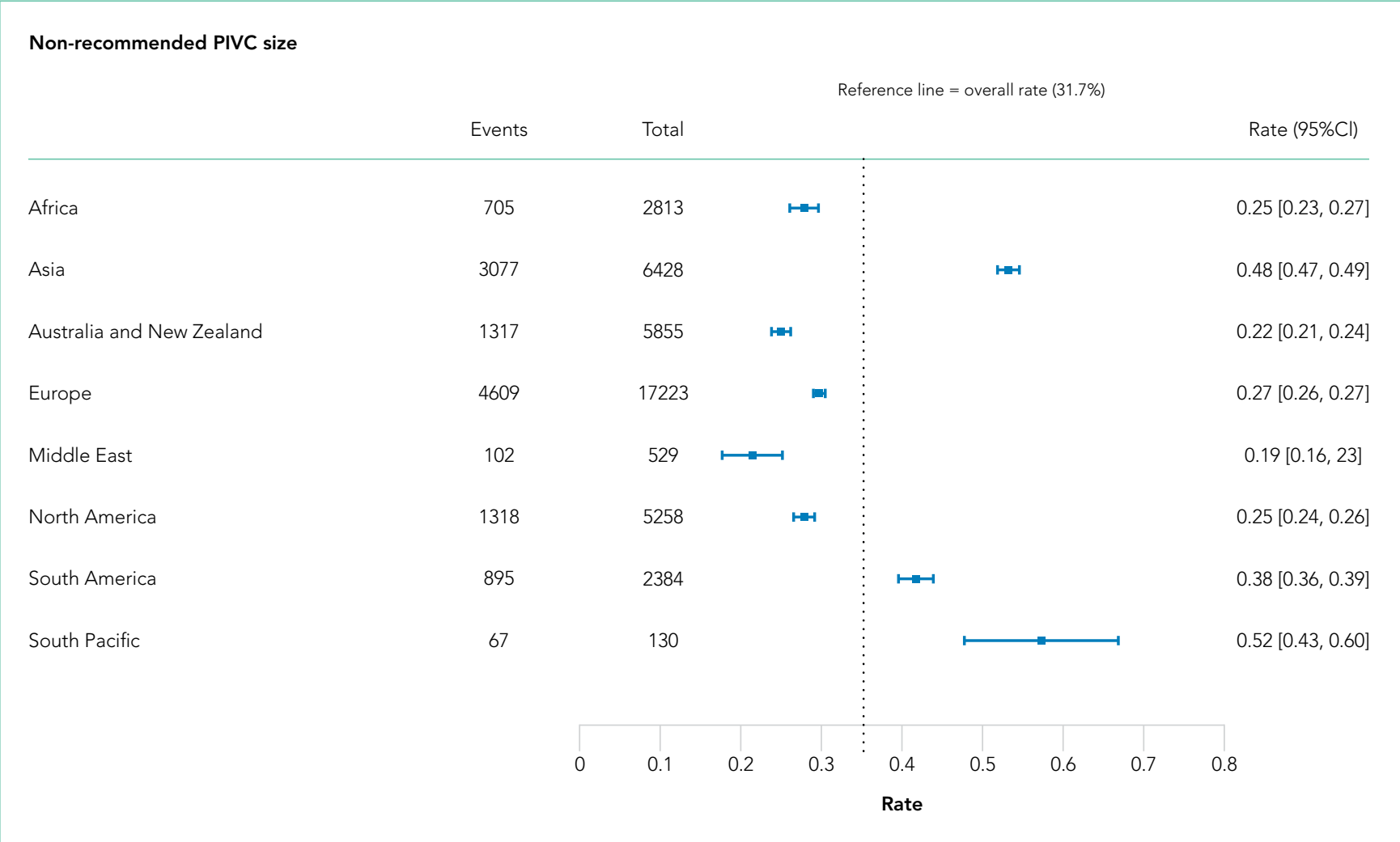

FIG 2. Non-Recommended Peripheral Intravenous Catheter size

optimal for PIVC insertion in adults, rather than areas of high flexion, because the forearm provides a wide surface area to secure and dress PIVCs. Forearm placement can reduce pain during catheter dwell as well as decrease the risk of accidental removal or occlusion., 31,27 We found only one-third of PIVCs were placed in the forearm, with most placed in the hand, antecubital veins, or wrist. This highlights an inconsistency with published recommendations and suggests that additional training and technology are required so that staff can better identify and insert PIVCs in the forearm for other than very short-term (procedural) PIVCp;s. ${ }^{19}$

Phlebitis triggering PIVC failure remains a global clinical challenge with numerous phlebitis definitions and varied assessment techniques. ${ }^{10}$ The prevalence of phlebitis has been difficult to approximate with varying estimates and definitions in the literature; however, it remains a key predictor of PIVC failure..$^{6,10}$ Identification of this complication and prompt removal of the device is critical for patient comfort and reducing CABSI risk. ${ }^{5,28}$ The overall prevalence of phlebitis signs or symptoms (defined in this study as having one or more signs of redness, swelling, or pain surrounding the insertion site) was just over $10 \%$, with pain and/or redness being most prevalent. These compromised PIVCs had not been removed as is recommended for such complications. ${ }^{19,28}$ Considering that our study was a snapshot at only one time point, the per-catheter incidence of phlebitis would be even higher; interestingly, among PIVCs with a documented insertion date and time, we observed that dwell time did not influence phlebitis rates.
Another concern is that nearly $10 \%(n=3,879)$ of PIVCs were malfunctioning (eg, leaking) but were still in place. To bring these problems into context, around 2 billion PIVCs are used annually worldwide; as a consequence, millions of patients suffer from painful or malfunctioning PIVCs staff had not responded. ${ }^{1,29}$ The placement of large-bore catheters, and smaller-gauge ones in adults, is known to increase the incidence of malfunction that leads to failure. There are a number of sound clinical reasons for the use of large-bore (eg, resuscitation and rapid fluid replacement) or small-bore (eg, difficult venous access with small superficial veins only visible and palpable) catheters. However, it would be expected that only a small proportion of patients would require these devices, and not one in three devices as we identified. This finding suggests that some PIVCs were inappropriate in size for general IV therapy and may reflect antiquated hospital policies for some clinical cohorts. $^{30,31}$

Overall, transparent dressings were used to cover the PIVC, but a number of patients were observed to have a sterile gauze and tape dressing ( $n=2,592,6 \%)$. Although the latter is less common, both dressing approaches are recommended in clinical practice guidelines because there is a lack of high-quality evidence regarding which is superior. ${ }^{21,22,32}$ Of concern was the use of nonsterile tape to dress the PIVC ( $n=5,169,12.7 \%)$. We found the prevalence of nonsterile tape use to be higher in lower-resourced countries in South America ( $n=714,30 \%)$, Africa ( $n=543,19 \%)$ and Europe $(n=3,056,18 \%)$ and this was likely related to institutional cost reduction practices. 
This finding illustrates an important issue regarding proper PIVC care and management practices in developing nations. It is widely known that access to safe health care in lower-resourced nations is challenging and that rates of mortality related to healthcare-associated infections are much higher. Thus, the differences we found in PIVC management practices in these countries are not surprising. ${ }^{33,34}$ International health networks such as the Infection Control Africa Network, the International Federation of Infection Control, and the Centers for Disease Control and Prevention can have great influence on ministries of health and clinicians in these countries to develop coordinated efforts for safe and sustainable IV practices to reduce the burden of hospital-acquired infections and related morbidity and mortality.

We found that $14 \%$ of all PIVCs had no documented IV medication or IV fluid administered in the previous $24 \mathrm{~h}$, strongly indicating that they were no longer needed. Australia/New Zealand, Europe, and North America were observed to have a higher prevalence of idle catheters than the remaining regions. This suggests that an opportunity exists to develop surveillance systems that better identify idle devices for prompt removal to reduce infection risk and patient discomfort. Several randomized controlled trials, a Cochrane review, and clinical practice guidelines recommend prompt removal of PIVCs when not required, if there are any complications, or if the PIVC was inserted urgently without an aseptic insertion technique. $21,28,35,36$ Idle PIVCs have been implicated in adverse patient outcomes, including phlebitis and CABSI. ${ }^{13,27}$

The substantial proportion of patients with a PIVC in this study who had no clinical indication for a PIVC, a symptomatic insertion site, malfunctioning catheter, and suboptimal dressing quality suggests the need for physicians, advanced practitioners, and nurses to adopt evidence-based PIVC insertion and maintenance bundles and supporting checklists to reduce the prevalence of PIVC complications. 19,21,38-40 Recommended strategies for inclusion in PIVC maintenance bundles are prompt removal of symptomatic and/or idle catheters, hand hygiene prior to accessing the catheter, regular assessment of the device, and replacement of suboptimal dressings. ${ }^{41,42}$ This approach should be implemented across all clinical specialties involved in PIVC insertion and care.

Our study findings need to be considered within the context of some limitations. The cross-sectional design prevented follow-up of PIVCs until removal to collect outcomes, including subsequent PIVC complications and/or failure, following the study observation. Ideally, data collection could have included patient-level preferences for PIVC insertion, history of PIVC use and/or failure, the number of PIVC insertion attempts, and the number of PIVCs used during that hospitalization. However, a cohort study of this magnitude was not feasible, particularly because all sites contributed staff time to complete the data collection. Only half of all initially registered sites eventually participated in the study; reasons for not participating were cited as local workload constraints and/or difficulties in applying for local approvals. Although efforts to enroll hospitals worldwide were exhaustive, our sample was not randomly selected but relied on self-selection and so is not representative, particularly for countries that contributed only one hospital site. Caution is also required when comparing inter regional differences, particularly developing regions, because better-resourced/ academic sites were possibly over represented in the sample. Nevertheless, PIVC variables differed significantly between participating hospitals, suggesting that the data represent a reasonable reflection of hospital variability.

\section{CONCLUSIONS}

On the basis of this international investigation, we report variations in the characteristics, management practices, and outcomes of PIVCs inserted in hospital patients from 51 countries. Many PIVCs were idle, symptomatic, had substandard dressings, and were inserted in suboptimal anatomical sites. Despite international best practice guidelines, a large number of patients had PIVCs that were already failing or at risk of complications, including infection. A stronger focus is needed on compliance with PIVC insertion and management guidelines; better surveillance of PIVC sites; and improved assessment, decision-making, and documentation.

\section{Acknowledgements}

We are extremely grateful to colleagues from across the globe who committed their time and effort to this study (for full details of countries and team members see Appendix 1).

Disclosures: Griffith University has received unrestricted investigator initiated research or educational grants on Claire M Rickard's behalf from product manufacturers 3M, Adhezion, Angiodynamics, Baxter, BBraun, Becton Dickinson, CareFusion, Centurion Medical Products, Cook Medical, Entrotech, Medtronic and Smiths Medical. Griffith University has received consultancy payments on Gillian Ray Burruel's behalf from manufacturers 3M, Bard; BD and Medline. Sheila Inwood has been a previous employee of CareFusion. Leonard Mermel has received research funding from Bard, and he has been a consultant for PuraCath, Marvao Medical, Bard and Applied Silver. Griffith University has received consultancy payments on Claire M. Rickard's behalf from manufacturers 3M, Bard, BBraun, BD, CareFusion, Mayo Healthcare, ResQDevices and Smiths Medical.

Funding Source: The authors wish to declare the OMG study has received unrestricted investigator-initiated research grants from Becton Dickinson (BD), CareFusion and 3M. B Braun provided funds for professional translation of data collection tools into several languages. All funds have been made payable to Griffith University or Western Sydney University and not to individual researchers.

\section{References}

1. Alexandrou E, Ray-Barruel G, Carr PJ, et al. International prevalence of the use of peripheral intravenous catheters. J Hosp Med. 2015;10(8):530-533. https:/doi.org/10.1002/jhm.2389

2. Zingg W, Pittet D. Peripheral venous catheters: an under-evaluated problem. Int J Antimicrob Agents. 2009;34(suppl 4):S38-S42. https:/ doi.org/10.1016/ S0924-8579(09)70565-5

3. Wallis MC, McGrail MR, Webster J, Gowardman JR, Playford G, Rickard CM. Risk factors for PIV catheter failure: a multivariate analysis from a randomized control trial. Infect. Control Hosp Epidemiol. 2014;35(1):63-68. https:/doi. org/10.1086/674398

4. Pujol M, Hornero A, Saballs M, et al. Clinical epidemiology and outcomes of peripheral venous catheter-related bloodstream infections at a university-affiliated hospital. J Hosp Infect. 2007;67(1):22-29.

5. Fakih MG, Jones K, Rey JE, et al. Sustained improvements in peripheral venous catheter care in non-intensive care units: a quasi-experimental con- 
trolled study of education and feedback. Infect. Control Hosp Epidemiol. 2012;33(5):449-455. https:/doi.org/10.1086/665322.

6. Helm RE, Klausner JD, Klemperer JD, Flint LM, Huang E. Accepted but unacceptable: peripheral IV catheter failure. J Infus Nurs. 2015;38(3):189-203. https:/ doi.org/10.1097/NAN.0000000000000100.

7. Austin ED, Sullivan SB, Whittier S, Lowy FD, Uhlemann AC. Peripheral intravenous catheter placement is an underrecognized source of Staphylococcus aureus bloodstream infection. Open Forum Infect Dis. 2016;3(2):ofw072. https:/ doi.org/10.1093/ofid/ofw072.

8. Stuart RL, Cameron D, Scott C, et al. Peripheral intravenous catheter-associated Staphylococcus aureus bacteraemia: more than 5 years of prospective data from two tertiary health services. Med J Aust. 2013;198(10):551-553.

9. Trinh $T T$, Chan PA, Edwards $O$, et al. Peripheral venous catheter-related Staphylococcus aureus bacteremia. Infect Control Hosp Epidemiol. 2011;32(6):579-583. https:/doi.org/10.1086/660099.

10. Ray Barruel G, Polit DF, Murfield JE, Rickard CM. Infusion phlebitis assessment measures: a systematic review. J Eval Clin Pract. 2014;20(2):191-202. https:/ doi.org/ 10.1111/jep.12107

11. Marsh N, Webster J, Flynn J, et al. Securement methods for peripheral venous catheters to prevent failure: a randomised controlled pilot trial. $J$ Vasc Access. 2015;16(3):237-244. https:/doi.org/10.5301/jva.5000348.

12. Carr PJ, Higgins NS, Cooke ML, Rippey J, Rickard CM. Tools, clinical prediction rules, and algorithms for the insertion of peripheral intravenous catheters in adult hospitalized patients: a systematic scoping review of literature. $J$ Hosp Med. 2017;12(10):851-858. https:/doi.org/ 10.12788/jhm.2836

13. Becerra MB, Shirley D, Safdar N. Prevalence, risk factors, and outcomes of idle intravenous catheters: An integrative review. Am J Infect Control. 2016;44(10):e167-e172. https:/ doi.org/10.1016/j.ajic.2016.03.073.

14. Robinson-Reilly M, Paliadelis $P$, Cruickshank M. Venous access: the patient experience. Support Care Cancer. 2016;24(3):1181-1187. https:/ doi. org/10.1007/s00520-015-2900-9.

15. Petroski A, Frisch A, Joseph N, Carlson JN. Predictors of difficult pediatric intravenous access in a community Emergency Department. J Vasc Access. 2015;16(6):521-526. https:/doi.org/10.5301/jva.5000411

16. Sou V, McManus C, Mifflin N, Frost SA, Ale J, Alexandrou E. A clinical pathway for the management of difficult venous access. BMC Nurs. 2017;16(1):64. https:/ doi.org/10.1186/s12912-017-0261-z

17. World Health Organization. Report on the burden of endemic health care-associated infection worldwide. Geneva2011. 9241501502.

18. Hirschmann H, Fux L, Podusel J, et al. The influence of hand hygiene prior to insertion of peripheral venous catheters on the frequency of complications. $J$ Hosp Infect. 2001;49(3):199-203. https:/doi.org/10.1053/jhin.2001.1077

19. Gorski L, Hadaway L, Hagle M, McGoldrick M, Orr M, Doellman D. Infusion therapy standards of practice. J Infus Nurs. 2016;39(suppl 1):S1-S159.

20. Abolfotouh MA, Salam M, Bani-Mustafa Aa, White D, Balkhy HH. Prospective study of incidence and predictors of peripheral intravenous catheter-induced complications. Ther Clin Risk Manag. 2014;10:993. https://doi.org/10.2147/ TCRM.S74685

21. Loveday H, Wilson J, Pratt R, et al. epic3: national evidence-based guidelines for preventing healthcare-associated infections in NHS hospitals in England. J Hosp Infect. 2014;86(suppl 1):S1-S70. https:/doi.org/10.1016/S0195$6701(13) 60012-2$

22. O'Grady NP, Alexander M, Burns LA, et al. Guidelines for the prevention of intravascular catheter-related infections. Clin Infect Dis. 2011;52(9):e162-e193. https:/doi.org/10.1093/cid/cir257

23. Cicolini G, Bonghi AP, Di Labio L, Di Mascio R. Position of peripheral venous cannulae and the incidence of thrombophlebitis: an observational study. J Adv Nurs. 2009;65(6):1268-1273. https:/doi.org/10.1111/j.13652648.2009.04980.x

24. Marsh N, Webster J, Larson E, Cooke M, Mihala G, Rickard C. Observational study of peripheral intravenous catheter outcomes in adult hospitalized patients: a multivariable analysis of peripheral intravenous catheter failure. $J$ Hosp Med. 2018;13(2):83-89. https:/doi.org/10.12788/jhm.2867.
25. One Million Global Catheters PIVC Worldwide Prevalence study. OMG study website http://www.omgpivc.org/. Accessed 23 March, 2017.

26. Von Elm E, Altman DG, Egger M, et al. The Strengthening the Reporting of Observational Studies in Epidemiology (STROBE) Statement: guidelines for reporting observational studies. Int J Surg. 2014;12(12):1495-1499. https:/doi. org/ 10.1136/bmj.39335.541782.AD

27. Fields JM, Dean AJ, Todman RW, et al. The effect of vessel depth, diameter, and location on ultrasound-guided peripheral intravenous catheter longevity. Am J Emerg Med. 2012;30(7):1134-1140. https:/doi.org/10.1016/j. ajem.2011.07.027.

28. Patel SA, Alebich MM, Feldman LS. Choosing wisely: things we do for no reason. Routine replacement of peripheral intravenous catheters. J Hosp Med. 2017;12(1):42-45

29. Newswire. Global Peripheral I.V. Catheter Market 2014 - 2018. New York, PR Newswire Assoc; 2014

30. Webster J, Larsen E, Booker C, Laws J, Marsh N. Prophylactic insertion of large bore peripheral intravenous catheters in maternity patients for postpartum haemorrhage: A cohort study. Aust N Z J Obstet Gynaecol. 2017.https:/ doi.org/10.1111/ajo.12759.

31. Rivera A, Strauss K, van Zundert A, Mortier E. Matching the peripheral intravenous catheter to the individual patient. Acta Anaesthesiol Belg. 2006;58(1):19.

32. Webster J, Gillies D, O'Riordan E, Sherriff KL, Rickard CM. Gauze and tape and transparent polyurethane dressings for central venous catheters. Cochrane Database Syst Rev. 2011;11:CD003827. https:/doi.org/10.1002/14651858. CD003827.pub2

33. Dieleman JL, Templin T, Sadat N, et al. National spending on health by source for 184 countries between 2013 and 2040. Lancet. 2016;387(10037):25212535. https:/ doi.org/10.1016/S0140-6736(16)30167-2.

34. Allegranzi B, Nejad SB, Combescure C, et al. Burden of endemic health-care-associated infection in developing countries: systematic review and meta-analysis. Lancet. 2011;377(9761):228-241. https:/ doi.org/10.1016/ S0140-6736(10)61458-4.

35. Rickard CM, Webster J, Wallis MC, et al. Routine versus clinically indicated replacement of peripheral intravenous catheters: a randomised controlled equivalence trial. Lancet. 2012;380(9847):1066-1074. https:/doi.org/10.1016/ S0140-6736(12)61082-4.

36. Webster J, Osborne S, Rickard CM, New K. Clinically indicated replacement versus routine replacement of peripheral venous catheters. Cochrane Database Syst Rev. 2015;8:CD007798. https://doi.org/10.1002/14651858. CD007798.pub4.

37. Yagnik L, Graves A, Thong K. Plastic in patient study: Prospective audit of adherence to peripheral intravenous cannula monitoring and documentation guidelines, with the aim of reducing future rates of intravenous cannula-related complications. Am J Infect Control. 2017;45(1):34-38. https:/doi. org/10.1016/j.ajic.2016.09.008

38. Boyd S, Aggarwal I, Davey P, Logan M, Nathwani D. Peripheral intravenous catheters: the road to quality improvement and safer patient care. J Hosp Infect. 2011;77(1):37-41. https:/doi.org/10.1016/j.jhin.2010.09.011.

39. DeVries $M$, Valentine $M$, Mancos P. Protected clinical indication of peripheral intravenous lines: successful implementation. J Assoc Vasc Access. 2016;21(2):89-92. https://doi.org/10.1016/j.java.2016.03.001

40. Rhodes D, Cheng A, McLellan S, et al. Reducing Staphylococcus aureus bloodstream infections associated with peripheral intravenous cannulae: successful implementation of a care bundle at a large Australian health service. $J$ Hosp Infect. 2016;94(1):86-91. https:/doi.org/10.1016/j.jhin.2016.05.020.

41. Rinke ML, Chen AR, Bundy DG, et al. Implementation of a central line maintenance care bundle in hospitalized pediatric oncology patients. Pediatr. 2012;130(4):e996-e1004. https:/doi.org/10.1542/peds.2012-0295.

42. Marshall J, Mermel L, Fakih M, Hadaway L, Kallen A, O'Grady N. Strategies to prevent central line-associated bloodstream infections in acute care hospitals: 2014 update. Infect. Control Hosp Epidemiol. 2014;35(suppl 2):S89107. https:/doi.org/10.1086/676533. 